Meta

Journal des traducteurs

Translators' Journal

\title{
Des traquenards de la version médicale
}

I. action, effect, potency et effectiveness

\section{Maurice Rouleau}

Volume 38, numéro 2, juin 1993

URI : https://id.erudit.org/iderudit/003999ar

DOI : https://doi.org/10.7202/003999ar

Aller au sommaire du numéro

Éditeur(s)

Les Presses de l'Université de Montréal

ISSN

0026-0452 (imprimé)

1492-1421 (numérique)

Découvrir la revue

Citer cet article

Rouleau, M. (1993). Des traquenards de la version médicale : i. action, effect, potency et effectiveness. Meta, 38(2), 268-274. https://doi.org/10.7202/003999ar d'utilisation que vous pouvez consulter en ligne.

https://apropos.erudit.org/fr/usagers/politique-dutilisation/ 


\section{DES TRAQUENARDS \\ DE LA VERSION MÉDICALE \\ I. ACTION, EFFECT, POTENCY \\ ET EFFECTIVENESS ${ }^{1}$}

MAURICE ROULEAU

Université du Québec à Trois-Rivières, Trois-Rivières, Canada

Il est reconnu que la traduction médicale exige de celui qui s'y adonne et s'y plaît une goût particulier pour la recherche terminologique. Personne ne peut, sans avoir fait au préalable une recherche poussée, traduire "complete blood count" par «formule sanguine complète» ou encore "chronic obstructive pulmonary disease" par «bronchopneumopathie chronique obstructive», sinon un familier du domaine.

Le traducteur médical, équipé de bons dictionnaires et rompu à cette discipline, ne se fait généralement pas piéger par de tels termes; leur aspect rébarbatif suffit normalement à attirer son attention sur la difficulté. Là où sa vigilance est trompée, c'est quand il rencontre des mots de langue générale utilisés dans un contexte de spécialité. Il se fait fort de pouvoir les traduire sans trop y réfléchir, car ces termes lui sont si familiers! Il ne se doute pas qu'il puisse y avoir une difficulté.

Pour illustrer la difficulté, inapparente mais réelle, que peuvent présenter des mots de langue générale, nous avons choisi d'examiner quatre termes: "action"2, "effect", "potency"» et "effectiveness", qui, tous, gravitent autour de la même idée, à savoir la raison de l'utilisation d'un médicament particulier. Même si ces termes, hors contexte, se décodent sans difficulté, leur traduction est souvent source d'erreurs.

Dans le cas des deux premiers termes ("action", "effect"), l'erreur vient du fait que le rédacteur, maîtrisant mal la notion des termes souvent construits en hypallage, utilise l'un pour l'autre et que le traducteur, maitrisant mal lui aussi les notions, colle au texte anglais. Dans le cas des deux derniers termes ("potency", "effectiveness"), l'erreur vient du fait que le traducteur, ne sachant pas très bien ce qui différencie la puissance d'un médicament de son efficacité, sera porté à confondre les notions et à croire qu'il s'agit de synonymes. Dans le premier cas, le traducteur ne voudra pas rendre "action" et "effect" par autre chose qu'«action» et «effet», alors qu'il devrait le faire; dans le second, il s'écarte du premier terme qui lui vient à l'esprit, alors qu'il ne le devrait pas.

$A C T I O N-E F F E C T$

Exemple 1: In vitro studies demonstrate that the bactericidal ACTION of $X Y Z^{3}$ results from inhibition of bacterial cell wall synthesis by inhibiting the transpeptidase and carboxypeptidase enzymes.

Dans une monographie de médicament, on commence généralement par présenter, sous la rubrique "ACTION"», la manière dont le médicament en question agit. Ce titre est habituellement rendu par «ACTION», quelquefois par MODE D'ACTION, dẹux équivalents tout à fait corrects. Influencé sans doute par la traduction qu'il vient de faire du terme "action", le traducteur se croit obligé de toujours le rendre par «action». C'est sans doute ce qui explique la traduction publiée : 
«Des études in vitro démontrent que l'action bactéricide de XYZ résulte de l'inhibition des enzymes impliqués dans la biosynthèse de la paroi cellulaire.»

traduction qui révèle un non-sens. En pharmacologie, «action» désigne le «mécanisme par lequel un médicament influence une entité biologique, que ce soit une molécule, une cellule, un organe ou un système», autrement dit la «manière dont un médicament agit». Quant à «effet», il désigne le «résultat d'une action, ce qui est produit par quelque chose» ou le «changement dans la fonction qui résulte d'une action (synonymes: réponse, résultat)». Il est évident que, si différentes fonctions sont commandées par un même mécanisme, la modification de ce dernier par un médicament déclenchera des phénomènes divers. Un médicament peut donc avoir une seule action et plusieurs effets. C'est le cas, par exemple, de l'atropine qui, en tant qu'antagoniste de l'acétylcholine au niveau des récepteurs post-ganglionnaires du système parasympathique, a des effets sur la sécrétion salivaire, le rythme cardiaque, le diamètre de la pupille, le tonus de l'utérus et de la vessie, la miction et les muscles lisses de l'intestin et des bronches.

Même si la distinction qui vient d'être faite se comprend facilement, la traduction de ces deux termes exige souvent un moment de réflexion parce que les notions qu'ils recouvrent ne sont pas précises à l'esprit du traducteur (souvent aussi à celui du rédacteur).

Tout traducteur qui se détache du texte et essaie de reformuler dans ses propres mots l'idée exprimée dans la langue de départ en arrivera immanquablement à dire que le médicament XYZ tue les bactéries parce qu'il empêche la synthèse de leur paroi cellulaire en bloquant l'activité de deux enzymes essentiels à ce processus; autrement dit, que l'inhibition des enzymes responsables de la biosynthèse de la paroi bactérienne entraîne la mort des bactéries. Cette reformulation permet d'identifier clairement l'action et l'effet du médicament. L'inhibition enzymatique est la manière d'agir du médicament, c'est donc son action, et la mort des bactéries est le résultat de l'action, c'est-à-dire son effet. Comme c'est l'effet qui résulte de l'action et non l'inverse, force est de reconnaître que la traduction proposée, qui fait résulter l'action de l'inhibition, est un non-sens. Cette confusion peut s'expliquer par le fait que, dans l'esprit du rédacteur, "bactericidal action" fait référence à l'effet du médicament (la mort des bactéries) plutôt qu'à son action qui a pour effet la mort, même si formellement le sujet du verbe "results" est "action" et non pas l'adjectif "bactericidal" et aussi par le fait que le traducteur ne se sent pas autorisé à traduire "action" par autre chose que «action".

En langage médical, l'association au mot action d'un adjectif désignant le résultat de l'action, par exemple action diurétique, action dilatatrice, action anorexigène, etc., est courante, aussi bien en français qu'en anglais, et elle n'est pas en soi fautive. Par exemple, dans la phrase suivante:

«... Certains dérivés de l'amphétamine ne sont utilisés que pour leur action anorexigène...» (Dictionnaire Flammarion 1989)

le syntagme «action anorexigène» ne crée aucun problème de compréhension. L'association n'est source de problème que si l'auteur l'utilise dans le sens de EFFET, comme cela est souvent le cas et notamment dans l'exemple 1. Une traduction correcte de cet exemple serait:

«Des études in vitro ont montré que le pouvoir bactéricide ${ }^{4} \mathrm{du} X \mathrm{XZ}$ s'explique par l'inhibition de la transpeptidase et de la carboxypeptidase, deux enzymes responsables de la biosynthèse de la paroi bactérienne.»

Bref, "action" ne se traduit pas obligatoirement par «action». Il faut toujours s'assurer, avant de traduire ce terme, que le rédacteur ne lui donne pas le sens de «effet». 
Exemple 2: Resistance: a quality that allows bacteria to survive [...] the EFFECTS of antibiotic medications.

L'idée véhiculée par cette phrase peut s'exprimer de diverses façons: les bactéries sont insensibles aux antibiotiques; l'effet des antibiotiques (raison pour laquelle ils sont utilisés) ne se manifeste pas; les bactéries ne meurent pas malgré la présence de l'antibiotique.

Est-il possible, compte tenu des définitions d'action et d'effet déjà mentionnées, que les bactéries survivent à l'effet d'un antibiotique? Si, comme dans l'exemple 1, l'action est l'inhibition des enzymes et l'effet la mort des bactéries, cela voudrait dire que les bactéries survivent à leur propre mort! Conclusion assez inattendue! Il ne serait pas plus correct de dire que les bactéries survivent à l'action de l'antibiotique, car cela voudrait dire que les enzymes sont inhibés, mais que les parois demeurent intactes. Compte tenu du rôle crucial joué par ces enzymes dans la biosynthèse de la paroi, un tel énoncé serait un non-sens. Ici, "effect" ne peut être traduit ni par effet ni par action. Une solution possible serait d'utiliser le mot «présence».

«Résistance: propriété que possède un microorganisme de survivre [...] en présence d'un antibiotique».

Ce problème de formulation n'est spécifique ni des antibiotiques ni de la langue anglaise. Dans le Flammarion, dictionnaire médical français, la définition de sympatholytique est la suivante:

«terme générique englobant les adrénolytiques et les ganglioplégiques, toutes substances qui inhibent, à des niveaux différents, les EFFETS de la stimulation sympathique.»

En fait, ce que les sympatholytiques font, c'est d'empêcher les médiateurs physiologiques d'exercer leur action. Ce faisant, ils préviennent l'apparition des effets de cette stimulation. Comme il n'est pas possible qu'un médicament inhibe les effets sans bloquer l'action, mieux vaut alors dire que c'est l'action qui est inhibée. C'est d'ailleurs ce que l'on trouve, dans le même dictionnaire, aux entrées adrénolytique et ganglioplégique.

Adrénolytique: $\quad$ «se dit de toute substance capable de diminuer ou de supprimer 1'ACTION de l'adrénaline sur les récepteurs $\alpha$ ou $\beta$ »

Ganglioplégique : «substance empêchant l'ACTION de l'acétylcholine...»

Bref, le terme "effect" ne se traduit pas obligatoirement par «effet». Il peut devoir se traduire par «action" ou par un autre terme plus pertinent.

Exemple 3: The ACTION of XYZ may last up to 6 hours and should last at least 4 hours. Consult your doctor immediately if the EFFECT lasts less than 3 hours.

Dans cet exemple, le problème est différent. Prises séparément, ces deux phrases ne présentent aucune difficulté; c'est leur juxtaposition qui gêne. La lecture consécutive de ces deux phrases donne l'impression que les termes "action" et "effect" sont synonymes, alors qu'en fait l'un est la raison d'être de l'autre. Pour éviter de problème d'apparente synonymie, mieux vaut utiliser deux fois le même terme. Dans le cas qui nous intéresse, «effet» serait préférable à «action», car ce que le patient est en mesure d'observer c'est l'effet du médicament. Une traduction honnête de l'exemple 3 serait par exemple :

«Il arrive que l'effet du XYZ dure 6 heures, mais il doit durer au moins 4 heures. Si jamais l'effet disparaît en moins de 3 heures, consultez immédiatement votre médecin.» 

français.

Ce problème d'apparente (ou de fausse) synonymie se rencontre également en

Exemple 3a: «... la théophylline exerce une ACTION dilatatrice sur les artères coronaires, pulmonaires et périphériques. Cet EFFET est bref et trop modéré pour ...» (Albingres et Tillement)

Si les auteurs utilisent «effet» comme mot charnière, c'est qu'ils pensent au résultat (la dilatation) et non à l'action (qui a pour effet de dilater). Cette ambiguïté peut pourtant être facilement levée :

«... la théophylline provoque la dilatation des artères coronaires, pulmonaires et périphériques. Cet effet est bref et trop modéré pour...»

Dans le dictionnaire Flammmarion, anticholinergique est défini ainsi :

Exemple $3 b$ : «substance contrariant l'ACTION de l'acétylcholine au niveau de la fibre postganglionnaire du système parasympathique, que cet EFFET s'exerce en inhibant la libération d'acétylcholine, ou en bloquant l'action de l'acétylcholine déjà libérée.»

Dans cet exemple, il n'est même pas possible de savoir à quoi fait référence l'auteur quand il parle d'effet, car ACTION est utilisé seul. La présence du mot effet est donc encore plus ambiguë. La définition aurait pu être formulée de façon à éviter ce problème :

«substance qui contrarie l'action de l'acétylcholine au niveau de la fibre post-ganglionnaire du système parasympathique, soit en empêchant l'acétylcholine d'être libérée, soit en empêchant l'acétylcholine déjà libérée d'exercer son action.»

Bref, «action» et «effet» ne sont pas synonymes. Quand ces deux termes se retrouvent dans deux phrases consécutives et que l'un d'eux sert de mot charnière, il y a une difficulté qui peut être résolue de différentes manières: utiliser deux fois le même terme (voir la traduction de l'exemple 3); rendre [action + adj. décrivant l'effet] par EFFET ou la nature de cet effet (voir l'exemple 3a); ou encore faire disparaître le mot effet mais définir l'action en question (voir l'exemple 3b).

Exemple 4: Expulsive EFFECTS such as coughing and sneezing serve to remove harmful substances from the body.

Quiconque oserait traduire cette phrase mot à mot se rendrait vite compte qu'elle n'a aucun sens. La toux et les éternuements sont loin d'être des effets ; ce sont eux qui ont pour effet d'expulser les substances de l'organisme. Mettre toux et éternuements en apposition à effets expulsifs, comme l'anglais le fait, n'a aucun sens. Il est évident que cette tournure s'est retrouvée sous la plume du rédacteur parce que, dans l'esprit de ce dernier, "expulsive effects" signifie expulsion, alors que grammaticalement toux et éternuements sont en apposition au substantif "action". Ce problème a déjà été rencontré à l'exemple 3a. L'auteur a utilisé le terme effet, pour rappeler ce dont il était question précédemment. Pour lui, action dilatatrice signifiait dilatation!

De plus, peut-on dire d'un effet qu'il est expulsif? NON, car, comme nous l'avons déjà dit, l'expulsion est elle-même l'effet en question. Expulsif signifiant «qui favorise ou accompagne l'expulsion", il est évident que ce n'est pas l'effet qui favorise l'expulsion. C.e qu'il faut comprendre, c'est que les effets [des réflexes] expulsifs (réflexes = toux et éternuements) consistent en l'élimination des particules dangereuses. En qualifiant ainsi l'effet d'expulsif, on attribue à un terme un qualificatif ne s'appliquant pas à ce terme mais à une notion que celui-ci implique. On utilise donc un adjectif en hypallage. Cette 
façon de dire n'est pas en soi condamnable, si la phrase n'est pas équivoque ni illogique. «Les effets mutagènes sont facilement mesurables de nos jours» se dit très bien, car cela se comprend sans problème. Cependant, dire les «effets expulsifs, tels que la toux et les éternuements» choque la raison. Dans un tel cas, il faut absolument débusquer le substantif qui est masqué dans l'hypallage (ici, c'est réflexes) et reporter l'idée d'effet plus loin dans la phrase si l'on veut que cette dernière ait un sens. Une traduction de l'exemple 4 pourrait être :

«Les réflexes expulsifs, que sont la toux et les éternuements, ont pour effet de chasser de l'organisme les substances dangereuses.»

Effet bactéricide, mentionné précédemment (voir note 4) est un autre exemple d'utilisation d'un adjectif en hypallage. Ce n'est pas l'effet qui tue les bactéries, mais bien le médicament. L'adjectif associé à effet est presque toujours en hypallage, exemple effet tératogène, effet mutagène, effet inflammatoire, etc. D'ailleurs, il ne s'agit pas d'un cas unique, les constructions en hypallage sont très fréquentes en langage médical (il suffit de penser à vaisseau sanguin, canal biliaire, diabète sucré, etc.). Même si ces constructions sont courantes, aussi bien en français qu'en anglais, leur traduction n'appelle pas nécessairement une construction identique dans la langue d'arrivée. Seule une analyse serrée du texte de départ peut justifier une telle utilisation.

Exemple 5: Mechanism of action of anti-pruritic drugs par Krause L. et Shuster S. [British Med J $22: 287$, 1984];

«Considérations générales sur la biochimie des hormones et leurs mécanismes d'action» par P.-H. Schmelck [Encycl Méd Chir (Paris) Glandes, Fasc. 10001 A10 (12-1978)]

Parler de «mécanisme d'action» est plutôt redondant. En effet, comme mécanisme signifie déjà à lui seul mode de fonctionnement, l'associer à action revient à dire «mode de fonctionnement de l'action»! Malgré sa bizarrerie, mécanisme d'action se rencontre de plus en plus sous la plume de scientifiques francophones. Ce phénomène s'explique sans doute par le fait que les textes scientifiques qui sont pour la plupart écrits en anglais et qui constituent la pâture quotidienne du scientifique francophone sont farçis de "mechanism of action" et que la préoccupation première du chercheur francophone qui publie n'est pas de sauver la langue française mais d'être lu et reconnu. Il ne s'interroge donc pas sur la pertinence de la formulation «mécanisme d'action». Elle trouve sa justification dans le fait qu'elle correspond littéralement à "mechanism of action" et que les deux mots qu'il utilise sont français. C'est ce que $\mathrm{j}$ 'appellerais de la contamination par fréquentation trop assidue.

Le traducteur ou le rédacteur francophones qui ne parviennent pas à s'exprimer autrement sont captifs des mots anglais; ils ne réussissent pas à aller chercher dans le fond d'expressions de leur langue maternelle celle qui répond à cette tournure anglaise, à savoir «mode d'action» que le petit Robert définit «forme particulière sous laquelle s'accomplit une action». Les articles de Krause et Shuster, et de Schmelck traitent respectivement du mode d'action des antiprurigineux et des hormones.

«Mécanisme» pourrait, compte tenu de sa signification, s'utiliser seul pour rendre "mechanism of action". Cependant, cette façon de dire ne serait pas idiomatique. En pharmacologie, on parle plus volontiers du mode d'action d'un médicament que du mécanisme d'un médicament. 


\section{POTENCY - EFFECTIVENESS5}

Même si, dans une monographie de médicament, ces deux termes sont moins utilisés que ACTION et EFFECT, ils n'en sont pas moins objets de méprise. Comme pour la première paire de termes étudiée, il n'est pas difficile de trouver les équivalents hors contexte de "potency" et de "effectiveness". C'est leur utilisation en contexte qui crée une difficulté pour le traducteur. La difficulté ne vient pas de l'anglais, comme cela était le cas avec la première paire de termes, mais plutôt de la non-maîtrise des notions françaises des équivalents. Le traducteur ne fait pas toujours la différence entre un médicament puissant et un médicament efficace.

On dit qu'un médicament est plus puissant qu'un autre si une plus petite dose du médicament est nécessaire pour produire le même effet. Par exemple, pour obtenir une diurèse satisfaisante, on peut utiliser soit $5 \mathrm{mg}$ de bendrofluméthiazide, $50 \mathrm{mg}$ d'hydrochlorothiazide ou $500 \mathrm{mg}$ de chorothiazide. Ces trois produits n'ont évidemment pas la même puissance. C'est le bendrofluméthiazide qui est le plus puissant, mais ils ont, aux doses mentionnées, la même efficacité ${ }^{6}$ : ils produisent un effet de même intensité. La méprise du traducteur vient du fait qu'il ne maîtrise pas les notions en cause. Il croît qu'un médicament efficace est puissant parce qu'un médicament puissant est nécessairement efficace. Il en vient donc à confondre ces deux attributs, à croire qu'ils sont synonymes. Il s'agit pourtant de deux notions bien différentes même si elles jouent sur le même clavier notionnel. Comme en anglais ces deux notions sont rarement confondues, "potency"» se traduira presque toujours par «puissance» et "effectiveness" par «efficacité».

\section{CONCLUSION}

Dans cet article, nous avons étudié quatre termes qui causent des problèmes au traducteur médical chargé de traduire une monographie de médicament.

Ces quatre termes ont un trait commun : chacun d'eux appelle presque uniquement un seul équivalent. Inconsciemment le traducteur en devient prisonnier. Il ne s'imagine pas qu'en contexte la situation puisse être différente. Il traduira donc toujours "action" par «action», "effect" par «effet», "potency" par «puissance» et "effectiveness" par «efficacité». À l'étape du peaufinage de son texte, le traducteur ne changera pas d'idée dans le cas des deux premiers termes (ce qu'il devrait faire dans bien des cas, comme nous l'avons montré), mais il se permet d'en changer dans le cas de la deuxième paire (ce qu'il ne devrait pas faire). Ce comportement s'explique par le fait que le traducteur et le rédacteur maîtrisent plus ou moins bien les notions que recouvrent ces quatre termes en langue de spécialité.

À ce problème notionnel vient se greffer, dans le cas des deux premiers termes, celui de l'utilisation de l'adjectif en hypallage. Le traducteur ou le rédacteur, en faisant appel à cette figure de style, confondent le substantif avec l'adjectif qui accompagne ce dernier. C'est ainsi qu'ils assimilent "bactericidal action" à l'effet (exemple 1), "expulsive effects" aux réflexes (exemple 4) ou encore «l'action dilatatrice» à la dilatation (exemple 3b).

Quand on traduit une monographie de médicament, il faut être très attentif à ces quatre termes, car ils cachent des difficultés. Pour pouvoir produire une traduction honnête des phrases contenant ces termes, il faut se rappeler leur définition :

ACTION :

EFFET : manière dont agit un médicament; mécanisme par lequel un médicament influence une entité biologique, que ce soit une molécule, une cellule, un organe ou un système. changement dans la fonction qui résulte d'une action (synonymes: réponse, résultat). 
PUISSANCE: $\quad$ pouvoir que possède un médicament de produire un effet.

EFFICACITÉ: $\quad$ 1) intensité de l'effet d'un médicament

ou

2) importance du succès thérapeutique.

\section{Notes}

1. Les prochains articles traiteront d'autres traquenards. Par exemple, est-ce que le mot "dosage" doit toujours être traduit par posologie? Est-ce qu'un comprimé est administré par voie orale ou par voie buccale ? Doiton dire un récepteur à histamine ou un récepteur d'histamine? Quels sont les principaux cooccurents prépositionnels de termes comme traitement, contre-indication, surdosage, etc.? Est-ce que le résultat d'un examen dont le suffixe est -graphie se termine obligatoirement par -gramme (radiographie-radiogramme, myélographie-... ? Comment s'affranchir de certaines structures anglaises, par exemple du "with" si fréquent en anglais?

2. Dans cet article, pour indiquer la langue d'origine du terme en question, nous utilisons les guillements anglais et les guillements français. "Action" est anglais et «action» français.

3. Les noms de médicaments dans cet article sont banalisés, car ce ne sont ni le produit ni la compagnie qui sont en cause, mais bien les mots utilisés.

4. Il serait plus idiomatique de dire EFFET BACTÉRICIDE (voir l'exemple 4).

5. Les rédacteurs de monographies de médicament utilisent indifféremment "efficacy" et "effectiveness".

6. Dans la littérature médicale, on parle d'efficacité d'un médicament pour traduire deux réalités différentes. D'une part, un médicament peut être plus efficace qu'un autre parce que l'intensité de son effet est plus grande. Tel est le cas de l'aspirine qui est plus efficace que l'acétaminophène (DCI : paracétamol) pour supprimer la douleur et l'inflammation dans les cas de polyarthrite rhumatoïde. D'autre part, une meilleure efficacité traduit aussi un succès thérapeutique chez un plus grand nombre de sujets. Les infections urinaires aiguës causées par $E$. coli seront traitées plus efficacement par le cotrimoxazole (association de sulfaméthoxazole et de triméthoprime) que par l'acide nalidixique.

\section{RÉFÉRENCES}

ALBINGRES E. et J.-P. TILLEMENT (1983) : «Médicaments de l'appareil respiratoire», Thérapeutique médicale, $2^{\mathrm{e}}$ édition, Paris, Jean Fabre, Flammarion Médecine Sciences.

Dictionnaire de médecine Flammarion (1989) : $3^{\mathrm{e}}$ édition, Paris, Médecine Sciences. 